\title{
Exploration of Colonic Looping Patterns in Undisturbed Cadaveric Specimens
}

Jacob Lam ${ }^{1}$, James Wilkinson ${ }^{1}$, Jonathan Brown ${ }^{2,3}$ Michelle Spear $^{4}$, Cecilia Brassett ${ }^{2}$

1) The University of Cambridge School of Clinical Medicine, CB2 OSP

2) Human Anatomy Teaching Group, Department of Physiology, Development and Neuroscience, University of Cambridge, CB2 3DY

3) Gloucestershire Hospitals NHS Foundation Trust, Gloucester, GL1 3NN

4) School of Anatomy, University of Bristol, Southwell Street, Bristol BS2 8EJ

\section{Exploration of Colonic Looping Patterns in Undisturbed Cadaveric Specimens}

\section{Abstract}

Introduction This study examines sex differences in the disposition of the sigmoid and transverse segments of the colon in undisturbed cadaveric abdomens and relates these findings to the anecdotal observation that colonoscopy is more challenging in females through the formation of tortuous bowel loops. Materials and Methods One hundred and twenty two undisturbed cadaveric abdomens were inspected. Three distinct configuration 
grades were separately assigned to the sigmoid and transverse segments of the colon on the basis of the pattern of the bowel loops observed in situ. Pearson's chi-squared test was used to analyze sex differences in bowel loop configuration and Spearman's rank correlation coefficient was calculated to identify co-occurrence of configuration grades in the subjects.

Results For the transverse segment, females had higher configuration grades corresponding to longer bowel loops with greater redundancy, compared to males $(p=0.000047)$. There was no sex difference in the sigmoid segment grade $(p=0.21636)$. Sigmoid and transverse segment grades were highly correlated in the subjects (coefficient=0.9994). Conclusion Sex differences in the configuration grades of the sigmoid or transverse colonic segments may be a significant contributing factor to increased difficulty of colonoscopy in females.

Keywords: colonic configuration; cadaveric; transverse colon; sigmoid colon; colonoscopy

\section{Introduction}

Colonoscopy is a common investigation and screening tool for detecting intraluminal colonic pathology. Implementation of the technique as part of bowel cancer screening programs has produced improvements in long-term prevention of deaths from colorectal cancer (Winawer et al., 1993; Zauber et al., 2012). Although this protective effect is most notable for distal lesions, detection of more proximal cancers has also been shown to be clinically important (Baxter et al., 2012). The rate of colonoscopy completion, which is defined as scope intubation of the caecum or terminal ileum, is considerably lower in females compared to males (Bowles et al., 2004). Thus, an inability to view the entire length of the colon during 
colonoscopy might lead to a failure to detect potentially lethal right-sided primaries, which are more common in females and carry poorer outcomes (Price et al., 2015).

Despite much of the colon being mobile within the peritoneal cavity, endoscopists can use the fixed segments as an anchor and apply external hand pressure or rotation of the patient to control looping and facilitate proximal intubation (Haycock et al., 2014). By week 12 of human embryogenesis the developing gut has completed a $180^{\circ}$ anticlockwise rotation and has returned to the peritoneal cavity from the umbilical hernia to give a right-sided caecum (Kim et al., 2003). The apposition of the dorsal mesentery to the posterior abdominal wall leads to fixation of the ascending and descending colons and a common misconception that these become retroperitoneal organs. Toldt's fascia of fusion preserves the affixed mesocolon (Culligan et al., 2012) in continuity with the posterior abdominal wall, leaving a variably mobile caecum, transverse and sigmoid colon within the peritoneal cavity. These structures can stretch or twist on their mesenteries during colonoscopy leading to pain and failure to progress. The 'alpha', 'reverse alpha' and 'N' loops are among several morphologically distinct sigmoid configurations that have previously been described (Haycock et al., 2014). Notably, the operator needs to employ a different combination of maneuvers to resolve the different types of loop, with each posing specific demands on the endoscopist's dexterity. Increasing mobility of the transverse colon can allow descent to the pubic symphysis to give a 'deep transverse' loop or twisting to give the 'gamma' loop, which is almost impossible to resolve. A mobile caecum requires the patient to be positioned in the right lateral for completion and visualization of the appendix orifice. Multiple factors have been suggested to explain why colonoscopy is more challenging in female patients. Uncontrolled colonic 'looping' is a common reason for abandoning a colonoscopy (Bowles et al., 2004) and this phenomenon is found to occur more frequently in females (Shah et al., 
2002). Left-sided loops are encountered more often in females (Anderson et al., 2000) and these are associated with the most requests for analgesia (Shah et al., 2002). Studies of colonic anatomy have suggested that female colons have greater propensity for the formation of more painful, tortuous loops due to increased redundancy. One such study using barium enemas found that females had greater total colonic lengths and longer transverse colons than males (Saunders et al., 1995). The former finding has since been confirmed with CT colonography (Khashab et al., 2009).

This study attempted to further elucidate sex differences in colonic anatomy, and consider how they might impact on the difficulty of colonoscopy. Our aim was to characterize the variation in the configuration of different colonic segments by inspection of their disposition in undisturbed cadaveric abdomens. Since it is recognized that challenging loops can be encountered in both sigmoid and transverse segments during colonoscopy we defined criteria to distinguish between three distinct grades of mobility for these regions. Colonic segments which followed a straight path with minimal redundancy were assigned lower grades, whereas increased redundancy with the formation of loops resulted in a higher grade. Further analysis aimed to investigate whether subjects with higher grades for the transverse colon would also have higher grades for the sigmoid segment. If this were the case, loop formation may be more extensive in such patients, resulting in more challenging colonoscopies.

The authors acknowledge that the anatomical factors that determine why colonoscopy is more difficult in females are likely to be complex and multifactorial. This study builds upon earlier work into sex differences in colonic lengths, although further investigation is required into the influence of other anatomical features, such as the abdominal musculature or the depth of the pelvis (Saunders et al., 1996), on passage of the scope through the colon. 


\section{Materials and Methods}

This study assessed variation in sigmoid and transverse colonic configurations in 122 undisturbed cadaveric abdomens. All cadavers had been embalmed using a mixture of formaldehyde, ethanol and methanol. The data were collected from 2014 to 2017 in the Human Dissection Room, Anatomy Building, Department of Physiology, Development and Neuroscience, at the University of Cambridge, UK. Sigmoid and transverse segments were identified by visual inspection and the disposition of the colon segments was documented with photographs and/or drawings. Colonic configurations were assessed and recorded immediately after the peritoneal cavity was opened and before the contents could be disturbed. A preliminary analysis of the observed dispositions of the sigmoid and transverse colons supported a novel classification system for mobility comprising three distinct configuration grades (I, II and III) for each segment (Tables 1 \& 2).

In a grade I sigmoid colon configuration, the entire segment is contained within the left iliac fossa, coursing from the descending colon to the rectum in a smooth curve (Fig 1A). For grade II, the sigmoid colon possesses a redundant loop which does not cross the midline (Fig 1B). In contrast, a grade III sigmoid configuration was identified as either forming multiple loops or possessing a long redundant loop which crossed the midline to the right iliac fossa (Fig 1C).

The transverse colon configuration was classed as grade I if it coursed from the splenic to hepatic flexure in a near-straight line (Fig 2A). In grade II, the transverse colonic segment looped inferiorly but the tip of the loop remained superior to the umbilicus (Fig 2B). In grade 
III, the transverse loop extended inferior to the umbilicus (Fig 2C), even reaching the pubic symphysis in a number of cases.

Exclusion criteria were applied to 12 subjects due to previous colonic resections or extensive adhesions secondary to intraabdominal surgery or malignancy. Statistical analysis was performed on the results obtained from the remaining 110 subjects using GraphPad Prism and Microsoft Excel software.

Student's t-test and Pearson's chi-squared test were used to test the null hypotheses that there was no sex difference in age at death or sigmoid and transverse colon configuration grades between males and females. A non-parametric form of Spearman's rank correlation coefficient was used to investigate whether subjects were likely to have the same configuration grade for both sigmoid and transverse segments.

\section{Results}

Of the 110 cadavers investigated, 52 were male (mean age 83.0, 95\% $\mathrm{Cl}[80.15,85.85]$ ) and 58 female (mean age $85.6,95 \% \mathrm{Cl}$ [83.08. 88.16]). This difference was not statistically significant $\mathrm{t}(108)=1.349, p=0.18$. The mobility results of the different colonic segments are shown in Table 3. There was a significant difference in the distribution of transverse colonic configurations between the sexes $(p=0.000047)$ (Fig 3$)$. Females were more likely to possess a grade III configuration at the transverse colon compared to males, whilst males 
were more likely to have a grade I configuration at the transverse colon. This sex difference was not observed in the sigmoid configuration grades $(p=0.21636)($ Fig 4$)$.

A strong correlation between sigmoid and transverse configuration grades was observed (Spearman's rank correlation coefficient, $\rho=0.9994$ ).

\section{Discussion}

This study has identified statistically significant differences in the configuration grades of the transverse colon between males and females in a cadaveric series of 110 subjects.

Previously, few studies have explored variation in colonic configuration in cadavers.

Furthermore, the literature contains conflicting results concerning sex differences in total colonic length, possibly due to the use of diverse investigative modalities (Phillips et al., 2015). We used a novel classification of colonic configuration in the transverse and sigmoid segments that could be consistently applied to all subjects. . Furthermore, consideration of the sigmoid and transverse segments as distinct entities provided a more refined analysis of locoregional anatomical variation.

The principal finding that female transverse colons have more extensive bowel loops than their male counterparts is corroborated by earlier studies using barium enemas (Saunders et al., 1995) and CT colonography (Khashab et al., 2009). This might contribute to an anatomical explanation for the observation that colonoscopy is technically more challenging in female patients (Waye et al., 1991; Church 1994; Saunders et al., 1996). Colons with greater redundancy might be more likely to form tortuous bowel loops during the procedure, which can be more difficult for the operator to resolve. This may result in difficulty in 
progression of the scope through the colon, and premature abandonment of the procedure, especially among less experienced colonoscopists, with important implications for clinical outcomes.

Interestingly, this study did not find a significant sex difference in sigmoid configuration grades. This is somewhat surprising in view of the increased prevalence of 'difficult' leftsided loops encountered in female patients during colonoscopy (Shah et al., 2002). It is possible that while bowel redundancy might have a role to play in the formation of a challenging loop, its overall perceived difficulty is influenced by other patient-specific anatomical factors, such as the pattern of sigmoid mesenteric attachments or the distribution of pain receptors.

Whilst visualization of the sigmoid colon in the undisturbed cadaver offers a useful estimate of its mobility, this does not necessarily reflect its latent capacity to distend as a result of insufflation during scope insertion. Future studies might benefit from technologies that can provide real-time magnetic imaging of the colonoscope configuration in order to relate descriptions of gross colonic anatomy with clinical findings (Lam et al., 2016).

This study also demonstrated that subjects were likely to have matching configuration grades for both sigmoid and transverse colons. Thus, transverse and sigmoid segments with greater looping were likely to co-exist in the same patients. This finding could be underpinned by a common mechanism, such as a generalized laxity of the mesentery or, conversely, a tendency for more extensive fixation to the posterior peritoneum. A cadaveric study by Phillips et al. (2015) found a high degree of variability in the portion of descending colon that was mobile. Intraoperative colonoscopy, a technique that has previously been used during resection of colorectal cancer (Brullet et al., 1992) as well as in the management 
of colonic hemorrhage (Berry, Campbell and Kettlewell 1988), is another investigative tool that could offer an insight into how difficult bowel loops might form during endoscopic examination.

One possible limitation of this study is the effect of embalming on colonic length and mobility, as the sigmoid colon, and especially its mesentery, may undergo considerable shrinkage (Bhatnagar et al., 2004). In this study, however, all comparisons were made between cadavers that have undergone the same embalming processes.

In summary, this is the first study to investigate sex differences in the configuration of transverse and sigmoid segments of the colon by the use of a novel classification system. Its key findings are that the transverse colon tends to have more extensive looping patterns in females compared to males, and that subjects with more extensive transverse colonic looping were also more likely to have a larger sigmoid segment loop. These findings provide an anatomical explanation for why colonoscopy may be more difficult in female patients . Further investigations should provide a greater insight into which clinical and anatomical factors lead to a more difficult colonoscopy, in order to allow identification of patients who may require a longer procedure or benefit from a more experienced endoscopist. 


\section{Acknowledgements}

The authors would like to express their gratitude to those who donated their bodies in order to enable this research project. All donors had provided consent for anatomical examination and research, including the publication of images before decease under the Human Tissue Act 2004

The authors have no conflicts of interest to disclose. 


\section{References}

Anderson, J., Gonzalez, J., Messina, C., \& Pollack, B. (2000). Factors that predict incomplete colonoscopy: thinner is not always better. The American Journal of Gastroenterology, 95(10), 27842787.

Baxter, N., Warren, J., Barrett, M., Stukel, T., \& Doria-Rose, V. (2012). Association Between Colonoscopy and Colorectal Cancer Mortality in a US Cohort According to Site of Cancer and Colonoscopist Specialty. Journal of Clinical Oncology, 30(21), 2664-2669.

Berry, A., Campbell, W., \& Kettlewell, M. (1988). Management of major colonic haemorrhage. British Journal of Surgery, 75(7), 637-640.

Bhatnagar, B., Sharma, C., Gupta, S., Mathur, M., \& Reddy, D. (2004). Study on the anatomical dimensions of the human sigmoid colon. Clinical Anatomy, 17(3), 236-243.

Bowles, C., Leicester, R., Romaya, C., Swarbrick, E., Williams, C., \& Epstein, O. (2004). A prospective study of colonoscopy practice in the UK today: are we adequately prepared for national colorectal cancer screening tomorrow? Gut, 53(2), 277-283.

Brullet, E., Montané, J., Bombardó, J., Bonfill, X., Noguè, M., \& Bordas, J. (1992). Intraoperative colonoscopy in patients with colorectal cancer. British Journal of Surgery, 79(12), 1376-1378.

Church, J. M. (1994). Complete colonoscopy: how often? And if not, why not? The American Journal of Gastroenterology, 89(4), 556-560. 
Culligan, K., Coffey, J., Kiran, R., Kalady, M., Lavery, I., \& Remzi, F. (2012). The mesocolon: a prospective observational study. Colorectal Disease, 14(4), 421-428.

Haycock, A., Cohen, J., Saunders, B., Cotton, P. B., \& Williams, C. (2014). Cotton and Williams' Practical Gastrointestinal Endoscopy. Wiley-Blackwell.

Khashab, M., Pickhardt, P., Kim, D., \& Rex, D. (2009). Colorectal anatomy in adults at computed tomography colonography: normal distribution and the effect of age, sex, and body mass index. Endoscopy, 41(8), 674-678.

Kim, W., Kim, H., Ahn, D., Kim, M., \& Park, H. (2003). Timetable for intestinal rotation in staged human embryos and fetuses. Birth Defects Research Part A-clinical and Molecular Teratology, 67(11), 941-945.

Lam, J., Wilkinson, J., Brassett, C., \& Brown, J. (2016). Analysis of Looping Patterns in Colonoscopy Using Scopeguide; Their Relation to Completion Times and Configurations In Cadaveric Colons. Gut, 65.

Phillips, M., Patel, A., Meredith, P., Will, O., \& Brassett, C. (2015). Segmental colonic length and mobility. Annals of The Royal College of Surgeons of England, 97(6), 439-444.

Price, T., Beeke, C., Ullah, S., Padbury, R., Maddern, G., Roder, D., ... Karapetis, C. (2015). Does the primary site of colorectal cancer impact outcomes for patients with metastatic disease. Cancer, 121(6), 830-835.

Saunders, B., Fukumoto, M., Halligan, S., Jobling, C., Moussa, M., Bartram, C., \& Williams, C. (1996). Why is colonoscopy more difficult in women. Gastrointestinal Endoscopy, 43(2), 124-126. 
Saunders, B., Halligan, S., Jobling, C., Fukumoto, M., Moussa, M., Williams, C., \& Bartram, C. (1995).

Can barium enema indicate when colonoscopy will be difficult. Clinical Radiology, 50(5), 318-321.

Shah, S., Brooker, J., Thapar, C., Williams, C., \& Saunders, B. (2002). Patient pain during colonoscopy: an analysis using real-time magnetic endoscope imaging. Endoscopy, 34(6), 435-440.

Waye, J., Yessayan, S., Lewis, B., \& Fabry, T. (1991). The technique of abdominal pressure in total colonoscopy. Gastrointestinal Endoscopy, 37(2), 147-151.

Winawer, S. J., Zauber, A. G., Ho, M. N., O'Brien, M. J., Gottlieb, L. S., Sternberg, S. S., . . Shike, M. (1993). Prevention of colorectal cancer by colonoscopic polypectomy. The National Polyp Study Workgroup. The New England Journal of Medicine, 329(27), 1977-1981.

Zauber, A., Winawer, S., O'Brien, M., Lansdorp-Vogelaar, I., Ballegooijen, M., Hankey, B., ... Waye, J. (2012). Colonoscopic Polypectomy and Long-Term Prevention of Colorectal-Cancer Deaths. The New England Journal of Medicine, 366(8), 687-696. 
Legends

Figure 1. Classification of sigmoid colon mobility
A. Grade I
B. Grade II
C. Grade III

Figure 2. Classification of transverse colon mobility.
A. Grade I
B. Grade II
C. Grade III 
Tables

\begin{tabular}{|l|l|}
\hline Sigmoid colon & Definition \\
\hline I & Follows the curve of the left iliac fossa into the pelvis. \\
\hline II & Forms a redundant loop that does not cross the midline. \\
\hline III & Has multiple redundant loops or a long loop that crosses the \\
& midline. \\
\hline
\end{tabular}

Table 1. Definition of sigmoid colon configuration grades.

\begin{tabular}{|l|l|}
\hline Transverse colon & Definition \\
\hline I & \\
\hline II & Direct course from splenic to hepatic flexure. \\
\hline III & Forms a loop that remains superior to the umbilicus. \\
\hline
\end{tabular}

Table 2. Definition of transverse colon configuration grades.

\begin{tabular}{|l|r|r|r|r|}
\hline \multirow{2}{*}{ Mobility } & \multicolumn{2}{|c|}{ Sigmoid } & \multicolumn{2}{c|}{ Transverse } \\
\cline { 2 - 5 } & Male & Female & Male & Female \\
\hline Low & 22 & 20 & 27 & 11 \\
\hline
\end{tabular}




\begin{tabular}{|l|r|r|r|r|}
\hline Intermediate & 15 & 26 & 18 & 18 \\
\hline High & 15 & 12 & 7 & 29 \\
\hline Total & 52 & 58 & 52 & 58 \\
\hline
\end{tabular}

Table 3. Sex differences between mobility of colonic segments. 

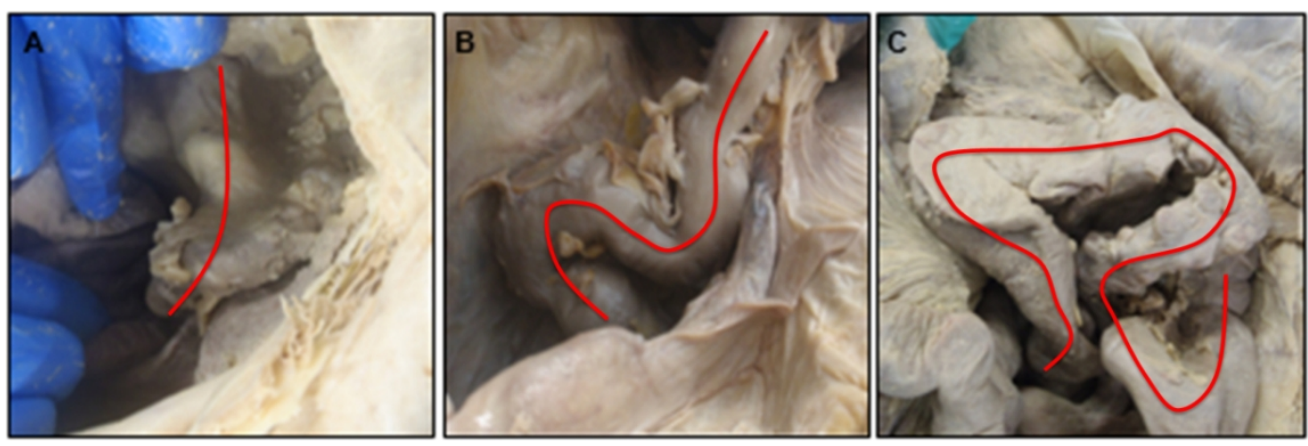

Figure 1. Classification of sigmoid colon mobility

A. Grade I

B. Grade II

C. Grade III 


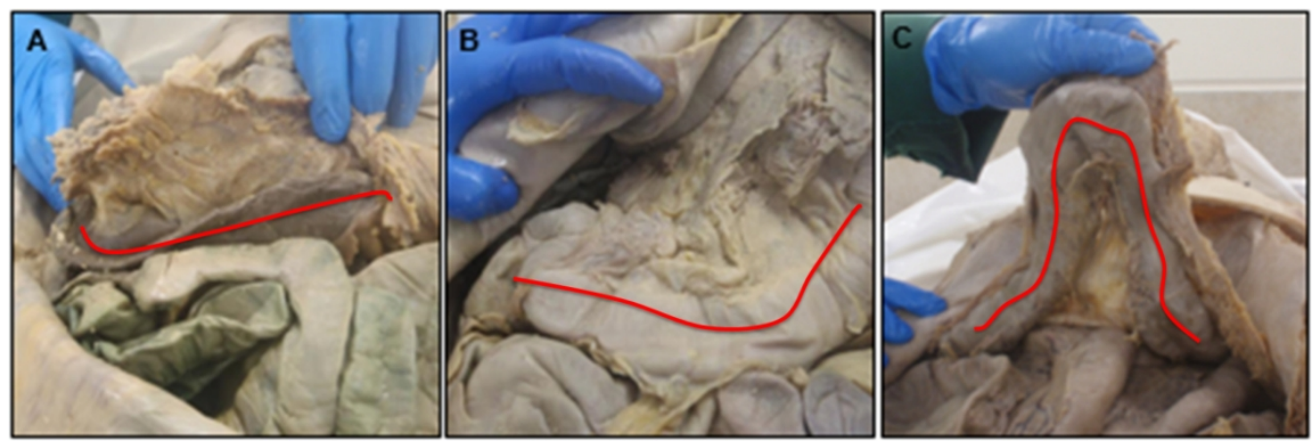

Figure 2. Classification of transverse colon mobility.

A. Grade I

B. Grade II

C. Grade III 


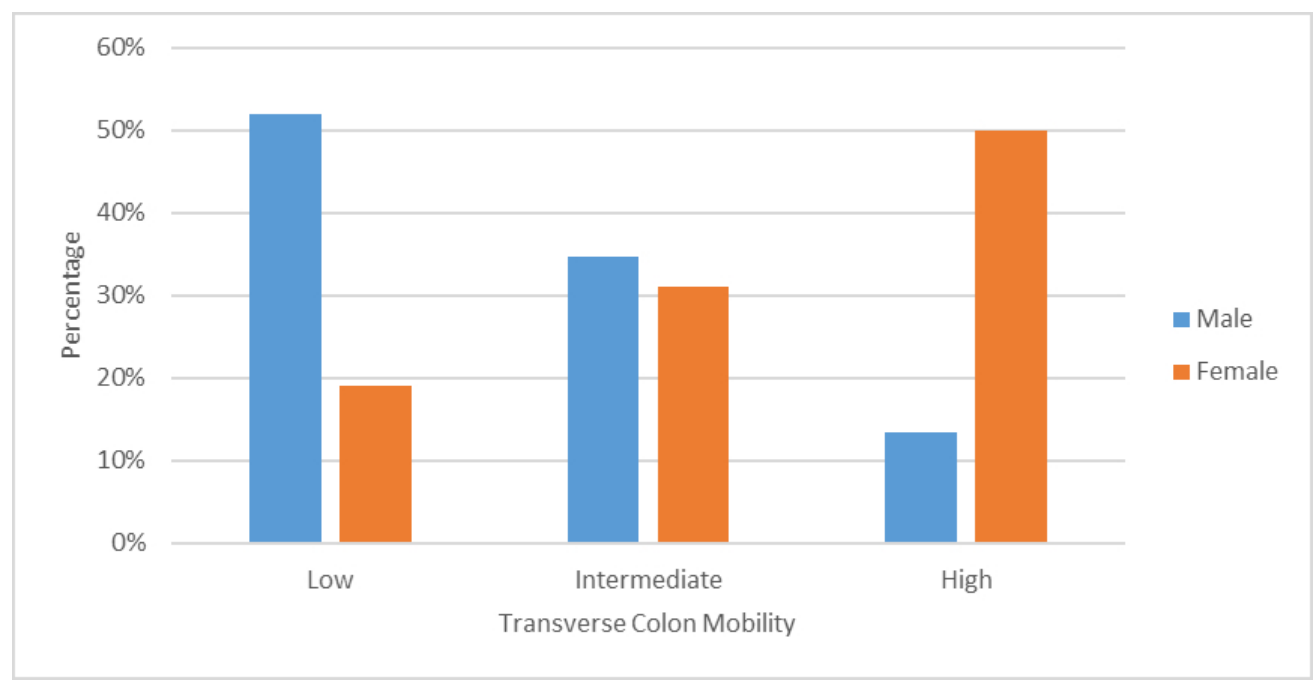

Distribution of transverse colonic configurations in males and females 


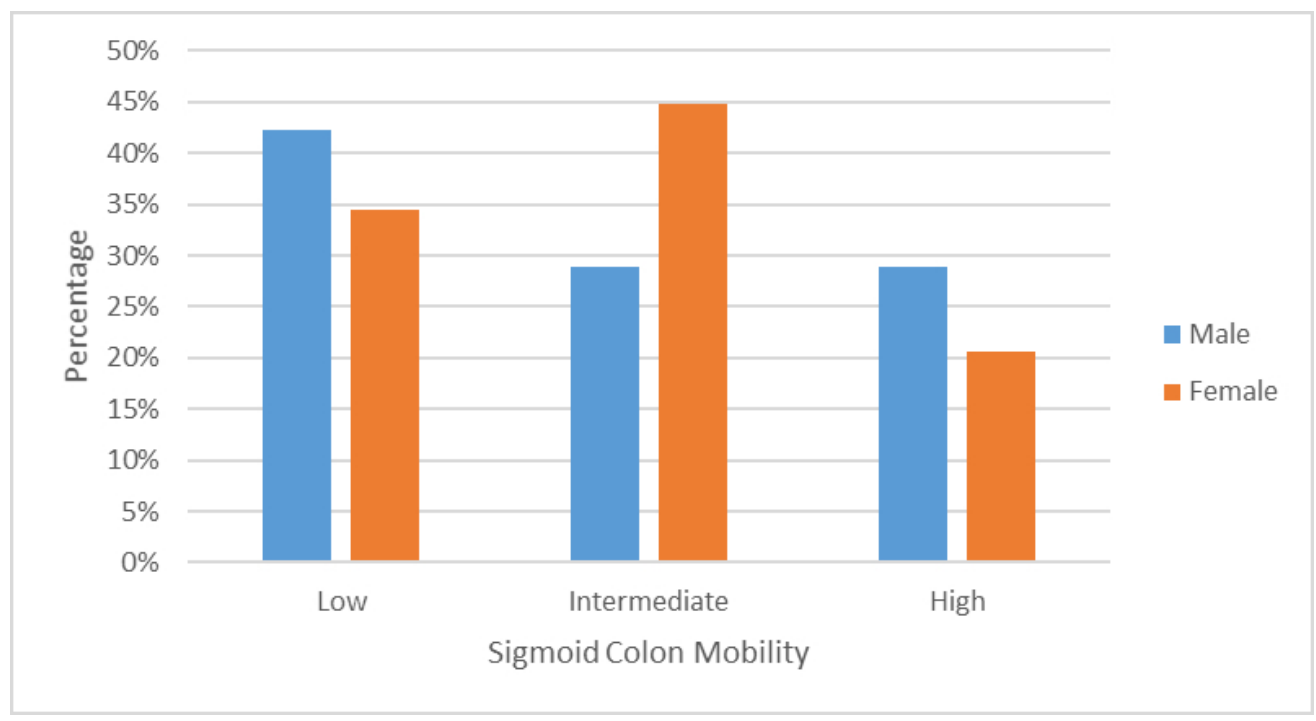

Distribution of sigmoid colonic configurations in males and females 